\title{
The frequency of polycystic ovary syndrome in young reproductive females in Qatar
}

\author{
This article was published in the following Dove Press journal: \\ International Journal of Women's Health \\ 16 December 2016 \\ Number of times this article has been viewed
}

\author{
Elham Sharif' \\ Sumaya Rahman' \\ Yumna Zia' \\ Nasser M Rizk ${ }^{1,2}$ \\ 'Biomedical Sciences Department, \\ College of Health Sciences, Qatar \\ University, Doha, Qatar; ${ }^{2}$ Physiology \\ Department, Mansoura Faculty of \\ Medicine, Mansoura University, \\ Mansoura, Egypt
}

\begin{abstract}
This was a prospective cross-sectional study in which 126 female students between the ages of 18 and 30 years were evaluated for the frequency of polycystic ovary syndrome (PCOS) through clinical interview, questionnaire, and anthropometric measurements. The diagnostic criteria of the US National Institutes of Health criteria were used. Menstrual irregularities (MI) were identified, and clinical hyperandrogenism was evaluated by self-assessment of hirsutism using modified Ferriman-Gallwey score. Blood analysis was done for measurement of prolactin, thyroid-stimulating hormone, and the androgen hormones. Of all the students, 37 (30.8\%) had MI, 38 (31.7\%) had clinical hirsutism, 37 (30.8\%) had acne, and $76(63.3 \%)$ had a family history of type 2 diabetes. The estimated frequency of PCOS was $18.33 \%$ according to the US National Institutes of Health definition. Hormonal analysis demonstrated a significant increase in androgens (total testosterone, dehydroepiandrosterone sulfate, and free testosterone), and a significant decrease in sex hormone-binding globulin in our PCOS group, with a $P$-value $<0.05$. This study revealed a higher level of the androgen hormones among PCOS subjects with a frequency of PCOS (18.33\%) similar to the global estimates of $10 \%-20 \%$.

Keywords: polycystic ovary syndrome, PCOS, hyperandrogenism, HA, hirsutism, menstrual irregularities, MI, frequency, hormonal profile, phenotype, Qatar, biochemical profile
\end{abstract}

\section{Introduction}

Polycystic ovary syndrome (PCOS) is the most prevalent endocrine disorder worldwide that affects $6 \%-8 \%$ of women. ${ }^{1}$ Enlarged ovaries, chronic anovulation, irregular menstrual cycles, androgen excess, and insulin resistance with consequences of acne, hirsutism, and infertility problems are main characters of PCOS. ${ }^{2}$ Studies in PCOS show that there is considerable heterogeneity in PCOS females, and hence, the need for a set of criterion to diagnose PCOS emerged. ${ }^{3,4}$ Several criteria are currently used for the diagnosis and definition of PCOS, such as the US National Institutes of Health (NIH) criteria, ${ }^{5}$ the Rotterdam criteria, ${ }^{6}$ and the Androgen Excess Society criteria. ${ }^{7}$

There are currently no comparable data about the phenotype profile, frequency of PCOS, and its features among young women in Qatar. Furthermore, awareness of PCOS prevalence has increased recently with the realization that this syndrome involves far more than the reproductive system and has medical consequences on metabolic aspects (such as metabolic syndrome, obesity, dyslipidemia, and insulin resistance), endocrine disorders (such as diabetes), and cardiovascular diseases (such as hypertension and atherosclerosis). In addition, it affects the psychosomatic perspective in females with PCOS, by causing obstetric and gynecological problems, such as infertility and endometrial cancers. Infertility problems have an influence on the social life of a human being, especially females. The aim of this study was to determine the frequency of PCOS defined by the NIH criteria, and its features
Correspondence: Elham Sharif Biomedical Sciences Department, College of Health Sciences, Qatar University, PO Box 2713, Doha, Qatar Tel +97444034788 Email e.sharif@qu.edu.qa
International Journal of Women's Health 2017:9 1-10

(c) (i) (2) () 2017 Sharif et al. This work is published and licensed by Dove Medical Press Limited. The full terms of this license are available at https://www.dovepress.com/terms.php

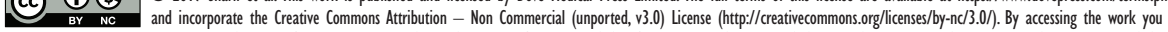
hereby accept the Terms. Non-commercial uses of the work are permitted without any further permission from Dove Medical Press Limited, provided the work is properly attributed. For permission for commercial use of this work, please see paragraphs 4.2 and 5 of our Terms (https://www.dovepress.com/terms.php).
for 
among the female students at Qatar University (QU). Our ultimate goal is to increase the awareness of PCOS and to promote effective early medical intervention and healthy lives for women.

\section{Subjects and methods Study subjects}

A population of 126 female students were recruited for the study as representative of the young females of Qatar. The total number of students approached was 130 , but after explanation, only 126 students decided to participate in the study. The participants were an unselected group of students, and all females were invited to take part in the study, regardless of having any symptoms of PCOS. Six subjects were excluded due to insufficient blood samples, incomplete surveys, and use of devices for hair removal. The final study sample consisted of 120 eligible participants, and only one of them was married and had infertility problems, while the remaining were single. Therefore, a cross-sectional study was performed on the 120 random adult female QU students. A campaign called "PCOS study in Qatar University" was initiated that aimed at screening the female population for hormonal irregularities and those who exhibited any symptoms suggestive of PCOS. Personal communication, flyers, posters, and social media (Facebook and Twitter) were used to recruit the study subjects. The study subjects were recruited from April 2011 until December 2011. The age group of the participants was between 18 and 30 years, at least 2-3 years after menarche to avoid any irregularity in the menstrual cycle. The participants were interviewed by the principal investigators and thereafter were examined in the university clinic by the medical team to check their medical condition. The study was approved by QU's Institutional Review Board (IRB) (QU-IRB; 58-E/11). All participants signed the written consent form after explanation of the study, and they received a copy of the ethical approval document.

\section{Definitions}

PCOS was diagnosed based on the NIH criteria ${ }^{5}$ which define a PCOS case as having the following:

Oligomenorrhea or amenorrhea, and clinical and/or biochemical evidence of hyperandrogenism with the exclusion of other conditions that can be involved in menstrual irregularities and hirsutism. ${ }^{5}$

The following clinical presentations, and biochemical profile, were used to identify subjects with suspected
PCOS: 1) menstrual irregularities (MI) such as oligomenorrhea, anovulation (defined as menstrual cycles $>35$ days and/or with $\leq 8$ cycles/year), and/or the presence of chronic amenorrhea, ${ }^{1}$ and 2) clinical hyperandrogenism (HA) (ie, hirsutism, alopecia, acne, and/or a modified FerrimanGallwey [mFG] score $\geq 17$ ) and/or subclinical features, with biochemical HA that is evident by an increase in serumfree testosterone in adult females above the normal level of $>0.663 \mathrm{nmol} / \mathrm{L}$, and $\mathrm{mFG}$ score defined as the extent of hair growth in a male pattern on 19 different body parts of a woman, measured on a severity scale of four. ${ }^{8}$ The NIH criteria were selected as they are used extensively for screening and in epidemiological studies, due to the lack of an ultrasound device in the university clinic, and because the majority of students who were single refused to go through clinical gynecological examination. The inclusion criteria were as follows: age 18-30 years, menarche from 10 to 15 years, no history of anatomical deformity, no use of tablets or hair-removal methods, normal prolactin and thyroid-stimulating hormone (TSH) levels, and a history of MI, such as oligomenorrhea and amenorrhea. Diagnosis of idiopathic hirsutism (IH) is one of the exclusion criteria used in this study, in which ovulatory dysfunction, HA, and other defined androgen excess disorders are ruled out. In the current study, subjects with a normal level of testosterone who used hair removal methods such as laser and/or dermatological treatment to improve hirsutism were considered to have $\mathrm{IH}^{9}{ }^{9}$

\section{Study protocols}

\section{Anthropometric measurements, questionnaire, and} Ferriman-Gallwey assessment

All participants went through anthropometric examinations, including weight and height; body mass index (BMI) was calculated by dividing the weight $(\mathrm{kg})$ by the square meters of height $\left(\mathrm{m}^{2}\right)$, and obesity was defined if BMI was $\geq 30 \mathrm{~kg} / \mathrm{m}^{2}$. All participants filled out a questionnaire providing details of their general demographic information (such as age and nationality) and medical history, including specific questions regarding known diagnosis of PCOS, diabetes, hypertension, cardiovascular disease, and family history of these conditions. The questionnaire also requested answers about menstrual history and lifestyle, including pharmaceutical history (Figure S1). The mFG was self-scored after explanation and demonstration of full details to all participants. A score $>17$ was considered HA as previously published among women of the same ethnic background in Arab Gulf States. ${ }^{10}$ 


\section{Biochemical assay}

Overnight fasting blood samples were drawn, followed by centrifugation, and serum was collected into aliquots and stored at $-80^{\circ} \mathrm{C}$ for further hormonal assay. Blood glucose was measured by an enzymatic colorimetric assay in the clinical chemistry laboratory at QU. Hormonal tests, including TSH, prolactin, insulin, testosterone, dehydroepiandrosterone sulfate (DHEAS), and sex hormone-binding globulin (SHBG), were conducted in the Chemistry Laboratory at Hamad Medical Corporation, Doha, Qatar. The principle of the assay used was chemiluminescent microparticle immunoassay (CMIA) for the quantitative determination of the following hormones: testosterone, DHEAS, insulin, TSH, prolactin, and SHBG in human serum. The free testosterone was calculated from total testosterone levels, albumin, and SHBG using a specified formula that has been used as a diagnostic tool for HA. ${ }^{11}$ Free androgen index (FAI) was also calculated as the total testosterone level in nmolL divided by the SHBG level in $\mathrm{nmol} / \mathrm{L}$ and then multiplied by 100 . FAI $>4.5$ was considered as a marker of biochemical HA. ${ }^{12}$

The interassay coefficient of variations $\%$ of the measured hormones was SHBG $\leq 10 \%$, TSH $\leq 10 \%$, DHEAS $\leq 10 \%$, testosterone $\leq 10 \%$, prolactin $\leq 6 \%$, and insulin $\leq 10 \%$. The cutoff values of the Endocrine Laboratory at Hamad Medical Corporation were used in this study (Table S1). TSH and prolactin were measured to exclude cases of MI caused by hyperprolactinemia and thyroid disturbances.

\section{Power calculation}

In the year 2011, there were 6,000 QU students, of whom $\sim 50 \%$ were Qatari, and nearly $50 \%$ of the Qatari students were females $(1,500)$.

Previous publications indicate that prevalence $(p)$ of PCOS is $0.05-0.10$ setting of the confidence interval $(Z)$ is 0.55 , and precision $(d)$ is 0.2 , so we needed to study 118 out of 2,000 subjects to be able to reject the null hypothesis with probability (power) of 0.8 . The type I error probability associated with this test of null hypothesis is 0.05 . This percentage represents a good portion as a sample size of young females in Qatar, which is a country with a small population.

\section{Statistical analysis}

Data were explored for outliers, skewness, and normality and transformed when necessary if normality assumption was violated. Continuous data are expressed as mean \pm SD for normally distributed variables, median and interquartile range
(25\%-75\%) for nondistributed continuous data, and number and (percentage) for categorical data. Two Student's $t$-tests and nonparametric Mann-Whitney test evaluated the differences between continuous variables, and two-independent samples $t$-tests were used accordingly for analysis. Chisquare test evaluated differences between categorical variables. The two-tailed $P \leq 0.05$ was considered as the cutoff value for significance. All statistical analyses were carried out using IBM SPSS Statistics 22.0 for Mac XP (IBM Corporation, Armonk, NY, USA) and GraphPad prism (Version 6, for Mac; GraphPad Software, La Jolla, CA, USA).

\section{Results}

The demographic, clinical and biochemical, and hormonal characteristics of the study subjects based on their clinical presentations of PCOS

Table 1 shows the comparison of the median and the interquartile values of the different variables for all study subjects, and further, between the control (non-PCOS) and the PCOS groups, among the study subjects. All the participants were within the age group range of 18-30 years, with a median age of 21 years. The participants' median and interquartile range of BMI was $22.95(19.95-25.81) \mathrm{kg} / \mathrm{m}^{2}$, and $11.6 \%$ of the participants were obese. No significant difference in age, BMI, age at menarche, and blood glucose was detected between non-PCOS and PCOS groups as listed in Table 1. The PCOS group exhibited significantly higher frequencies of $\mathrm{MI}$ and hirsutism than the non-PCOS group, with a $P$-value of $<0.05$, as listed in Table 1. Clinical hirsutism assessed by the "mFG score" was found in 38 cases of the study, of whom 16 had IH. Among the hormones assayed, the total testosterone, DHEAS, free testosterone, and FAI are significantly higher in PCOS subjects than in non-PCOS subjects $(P \leq 0.0001)$, as listed in Table 1. In contrast, no significant difference in the median concentrations of SHBG, insulin, prolactin, and TSH was detected between the two groups.

\section{The frequency of PCOS among the study subjects and the risk factors for PCOS}

Of the 120 study subjects, 22 students met the NIH criteria for PCOS diagnosis, resulting in a frequency of $18.33 \%$, and their main diagnostic criteria are listed in Table S2. All PCOS cases had oligo-anovulation and clinical/biochemical HA (100.0\% had hirsutism with a score of $\geq 17.0,63.6 \%$ had acne, and $83.4 \%$ had abnormally elevated FAI, but none had abnormal free testosterone level). Table 2 shows the relative 
Table I Clinical and biochemical characteristics of all study subjects, non-PCOS and PCOS

\begin{tabular}{|c|c|c|c|c|}
\hline Variables & All study subjects & Non-PCOS (n=98, $81.67 \%)$ & $P \cos (n=22,18.33 \%)$ & $P$-value \\
\hline Age, median (IQR), years & $21.00(19.00-22.50)$ & $22.0(19.00-22.50)$ & $21.00(20.00-22.00)$ & 0.921 \\
\hline BMI, median (IQR), kg/m² & $22.95(19.95-25.81)$ & $23.37(19.95-24.96)$ & $23.93(19.90-28.48)$ & 0.155 \\
\hline Age of menarche, median (IQR), years & $12.00(11.00-13.00)$ & $12.00(11.00-13.00)$ & $12.00(12.0-13.0)$ & 0.915 \\
\hline Hirsutism, n (\%) (mFG score $>17$ ) & $38(31.7)$ & $16(16.3)$ & $22.0(100)$ & 0.028 \\
\hline Menstrual irregularities, n (\%) & $37(30.8)$ & $15(15.3)$ & $22(100)$ & 0.017 \\
\hline Acne, n (\%) & $37(30.8)$ & $23(23.5)$ & $14(63.6)$ & 0.023 \\
\hline Glucose, median (IQR), mg/dL & $88.00(81.50-97.50)$ & $87.00(82.00-96.00)$ & $90.50(80.00-101.50)$ & 0.701 \\
\hline Total testosterone, median (IQR), nmol/L & $1.39(0.77-2.28)$ & $1.30(0.60-1.79)$ & $2.70(2.04-3.45)$ & $<0.000$ I \\
\hline DHEAS, median (IQR), $\mu \mathrm{mol} / \mathrm{L}$ & $8.13(6.04-10.11)$ & $7.40(5.54-9.56)$ & $10.1(8.06-13.64)$ & $<0.000$ I \\
\hline SHBG, median (IQR), nmol/L & $51.00(45.00-62.00)$ & $51.00(37.50-67.00)$ & $45.00(28.00-62.00)$ & 0.0697 \\
\hline Free testosterone, median (IQR), $\mathrm{nmol} / \mathrm{L}$ & $0.023(0.008-0.036)$ & $0.017(0.013-0.020)$ & $0.043(0.037-0.049)$ & $<0.000 \mathrm{I}$ \\
\hline Insulin, median (IQR), mlU/L & $12.00(6.00-25.00)$ & $12.00(6.00-23.50)$ & $\mid 4.00(6.00-31.00)$ & 0.259 \\
\hline Prolactin, median (IQR), mIU/L & $320.5(225.50-457.50)$ & $312.00(211.0-47 \mid .0)$ & $361.00(266.0-401.0)$ & 0.195 \\
\hline TSH, median (IQR), mlU/L & $1.47(1.09-1.98)$ & $1.47(1.14-1.95)$ & $1.44(1.02-2.40)$ & 0.876 \\
\hline FAl, median (IQR) & $2.80(1.17-5.17)$ & $1.86(1.12-3.76)$ & $5.75(3.14-10.93)$ & $<0.0001$ \\
\hline
\end{tabular}

Notes: Descriptive characteristics obtained by Wilcoxon/Kruskal-Wallis tests with nonparametric comparison for each pair using Wilcoxon method. Categorized data were analyzed by chi-square test. Two-tailed $P$-value $<0.05$ is significant. Free testosterone was calculated by subtracting the testosterone bound to SHBG and albumin.

Abbreviations: BMI, body mass index; DHEAS, dehydroepiandrosterone sulfate; FAI, free androgen index; IQR, interquartile range; mFG, modified Ferriman-Gallwey; PCOS, polycystic ovary syndrome; SHBG, sex hormone-binding globulin; TSH, thyroid-stimulating hormone.

risk factors between the PCOS and the control subjects. Of interest, PCOS subjects have significantly higher frequencies of family history of PCOS, acne, and obesity than the control subjects with $P$-values $0.003,0.023$, and 0.0005 , respectively. Although PCOS subjects exhibited higher frequency of family history of diabetes and hyperinsulinemia, the difference was not significant when compared with the non-PCOS subjects $(P>0.05)$, as shown in Table 2 .

\section{The biochemical and hormonal features of subjects presenting with $\mathrm{MI}$}

This study highlights the median and interquartile values of the different hormone concentrations and FAI in all the study subjects with MI and their $P$-values, as listed in Table 3. Of interest, MI subjects with PCOS have significantly higher median values of free as well as total testosterone, DHEAS, and FAI than MI subjects with nonPCOS. No significant difference was found between the

Table 2 Relationship between possible risk factors and PCOS in the study subjects and after adjustment for the confounding factors

\begin{tabular}{llll}
\hline Risk factors & Non-PCOS & PCOS & P-value \\
\hline FH of PCOS, n (\%) & $8(8.2)$ & $9(40.9)$ & 0.003 \\
FH of diabetes, n (\%) & $55(56.1)$ & $21(95.5)$ & 0.156 \\
Acne, n (\%) & $23(23.5)$ & $14(63.6)$ & 0.023 \\
Hyperinsulinemia & $26(26.5)$ & $9(40.9)$ & 0.343 \\
$\begin{array}{l}\text { (>23.0 mIU/L), n (\%) } \\
\text { Obesity, n (\%) }\end{array}$ & $5(5.1)$ & $9(40.9)$ & 0.0005 \\
\hline
\end{tabular}

Notes: Data were analyzed by chi-square test for comparison between non-PCOS and PCOS groups, with their corresponding $P$-values. Odds ratio and $95 \% \mathrm{Cl}$ were analyzed by logistic regression with the adjustments of these factors with their $P$-value. Two-tailed $P$-value $\leq 0.05$ is significant.

Abbreviations: $\mathrm{FH}$, family history; PCOS, polycystic ovary syndrome. two groups for SHBG, TSH, prolactin, and insulin, with $P$-value $>0.05$.

\section{Discussion}

This study investigated the hormonal and phenotype profile and the frequency of PCOS among young female students in the age group of 18-30 years. The study highlighted important data about the frequency and the hormonal and phenotype profile in PCOS subjects as well as in subjects presenting with MI, among this young female study population. Such findings will be discussed in the following paragraphs.

The frequency of PCOS in this study was $18.33 \%$ according to the NIH criteria. PCOS prevalence depends on the recruitment process of the study population and the criteria used for its diagnosis and definition. Two studies on PCOS in Iran reported PCOS prevalence rates of 11.7 and $7 \%$ based on the NIH criteria, respectively. ${ }^{13,14}$ The frequency of PCOS by the NIH standards in a communitybased study in Sri Lanka was 6.3\% in women aged between 15 and 39 years. ${ }^{15} \mathrm{~A}$ recent assessment of the frequency of PCOS in North India was 3.7\% in women aged between 18 and 25 years. ${ }^{16}$ The frequency of PCOS in Caucasian women from Madrid, Spain, was $6.5 \% .{ }^{17}$ A recent study among Palestinian subjects aged 18-24 years reported a PCOS prevalence of $7.3 \% .{ }^{18}$ The discrepancy between the previous studies regarding the prevalence of PCOS and the data of this study could be attributed to the recruitment process of the study subjects, small sample size, age difference, and/or ethnic background. For example, the age group 
Table 3 Biochemical features of Ml subjects

\begin{tabular}{|c|c|c|c|c|}
\hline Laboratory test & All MI & MI (non-PCOS) & MI with PCOS & $P$-value \\
\hline Free testosterone $(\mathrm{nmol} / \mathrm{L})$ & $0.032(0.02 I-0.042)$ & $0.015(0.005-0.025)$ & $0.039(0.03 I-0.047)$ & 0.0002 \\
\hline $\mathrm{T}$ testosterone $(\mathrm{nmol} / \mathrm{L})$ & $2.04(0.92-2.7 I)$ & $0.91(0.70-1.29)$ & $2.61(2.03-3.06)$ & 0.0001 \\
\hline DHEAS $(\mu \mathrm{mol} / \mathrm{L})$ & $8.67(6.63-10.91)$ & $6.96(5.58-8.13)$ & $9.84(8.22-12.11)$ & 0.0026 \\
\hline SHBG (nmol/L) & $55.0(29.5-69.00)$ & $67.00(47.00-72.00)$ & $50.00(26.50-62.00)$ & 0.0580 \\
\hline FAI & $2.79(1.17-5.15)$ & $2.67(1.14-4.95)$ & $3.58(1.36-7.65)$ & $<0.0001$ \\
\hline TSH (mIU/L) & $1.28(1.07-2.19)$ & $1.24(0.99-1.47)$ & $1.36(1.08-2.49)$ & 0.516 \\
\hline Prolactin (mIU/L) & $331.00(273.00-395.00)$ & $324.00(283.00-372.00)$ & $360.50(269-395.76)$ & 0.465 \\
\hline Insulin (mIU/L) & $14.00(5.50-35.50)$ & $12.00(5.00-34.00)$ & $14.50(6.00-37.00)$ & 0.467 \\
\hline
\end{tabular}

Notes: Data are presented by median and IQR values (25\%-75\%). Data were calculated by nonparametric comparison for each pair using Wilcoxon method. Two-tailed $P$-value $<0.05$ is significant.

Abbreviations: DHEAS, dehydroepiandrosterone sulfate; FAI, free androgen index; IQR, interquartile range; MI, menstrual irregularities; PCOS, polycystic ovary syndrome; SHBG, sex hormone-binding globulin; T, total; TSH, thyroid-stimulating hormone.

of this study sample was narrow, including women aged 18-30 years, while some other studies included women aged 18-45 years to limit the phenomenon of irregular menstrual cycles in early age and premenopausal age. The recruitment of the study subjects was an unselected group of students. Due to the nature of the study, this could have encouraged some students experiencing PCOS-like symptoms to come forward for this study, while others without PCOS were not involved in PCOS screening, which could have had an impact on the results of this present study. On the contrary, previous studies have often used hospital settings such as blood donation centers ${ }^{19}$ and in vitro fertilization (IVF) clinic patients, ${ }^{20}$ who might be considered as biased samples. Females who donate blood are generally healthy, which could account for the low frequency of PCOS in those study groups. However, on the contrary, subjects recruited from IVF clinic patients desiring conception may have gynecological problems such as PCOS-like conditions, which may explain the high frequency of PCOS in those study groups. Similar concerns have been voiced by the United Arab Emirates (UAE) fertility clinics, that the frequency of PCOS in the Gulf region and South Asia appears to be much higher, though no exact figures are available. ${ }^{21}$ Also, the difference in the definition of HA may also account for the difference in the frequency of PCOS among different studies worldwide. Knochenhauer et $\mathrm{al}^{22}$ defined HA by Ferriman-Gallwey score only. In this study, HA was defined either clinically by evidence of hirsutism ( $\mathrm{mFG}$ score $\geq 17$ ), with acne, or biochemically by increased free testosterone level above the cutoff value $>0.663 \mathrm{nmol} / \mathrm{L}$ and FAI. ${ }^{23}$ Clinical hirsutism was found in $31.7 \%$ of all study subjects and $100.0 \%$ of PCOS subjects. In fact, we did not consider females with only acne to be hyperandrogenic because acne may be due to Propionibacterium. Different studies in Srilanka, ${ }^{15}$ Spain, ${ }^{17}$ and Palestine ${ }^{18}$ had utilized a different range for Ferriman-Gallwey score (hirsutism defined by a score of $\geq 8$ ). Meanwhile, we followed the model used by the neighboring countries of similar ethnic background, such as Saudi Arabia, Bahrain, and UAE, with a score of $\geq 17$. We found in this study that among the PCOS group, 100\% had clinical hirsutism. The frequency of facial hirsutism without PCOS remains unclear in this region of the world, although PCOS continues to be one of the most common causes of hirsitism. Previous studies in Arab Gulf countries showed that PCOS is the leading cause of hirsutism among women and reached $82 \%$ and $91 \%$ in Saudi Arabia and UAE based studies, respectively. ${ }^{10,11} \mathrm{IH}$ can not be solely excluded from the PCOS diagnosis since many women may report that they have regular periods despite oligoovulation. This finding further warrants studying ethnic diversity in androgenic manifestation.

In this study, of the 22 cases of PCOS, four (3.31\%) cases had been previously diagnosed with infertility problems and 18 new cases were defined, implying that $\sim 82 \%$ of the young women were unaware of their health condition. The same idea was echoed in a cohort study in Australia, which found that $68 \%-69 \%$ of the diagnosed females with PCOS were unaware of their status. ${ }^{25}$ Hence, the study also draws attention to the issues of many women with PCOS in the community who remain undiagnosed.

This study showed that having a family history of PCOS is a significant risk factor for developing PCOS $(P=0.0001)$. This idea is reflected in the literature claiming PCOS to be familial, ${ }^{26}$ and various factors may be differentially inherited. Ideally, circulating free testosterone is used as a marker for the detection of PCOS, ${ }^{23}$ but another study has shown that DHEAS also plays a significant role in causing HA and could be used as a biomarker for HA. ${ }^{27}$ This study revealed that high level of DHEAS is a significant finding in PCOS, which is consistent with a previous study. ${ }^{28}$ 
In females, the ovaries and adrenal glands are responsible for the production of androgens. According to a study by Azziz, ${ }^{1}$ total testosterone $>4.8 \mathrm{nmol} / \mathrm{L}$ is considered a sign of an androgen-secreting tumor, but none of our samples had a level $>4.8 \mathrm{nmol} / \mathrm{L}$. Also, another study showed that tumors with total testosterone slightly $>2 \mathrm{nmol} / \mathrm{L}$ were extremely rare (18 out of 400 cases). Actually, as in most previous studies, we were not able to control the collection of blood samples at the time of the menstrual periods of participants due to irregular periods in these PCOS subjects. Knochenhauer et $\mathrm{al}^{22}$ and Asuncion et $\mathrm{al}^{17}$ have discussed that none of these factors have a significant effect on the circulating levels of androgens.

In our population sample, only four females had type 1 diabetes, detected among the control subjects, and none had type 2 diabetes. However, elevated insulin levels were prevalent (41\%) in the PCOS group, but no cases were diagnosed with type 2 diabetes. A study conducted in Boston, MA, USA, reported that $7.5 \%$ of PCOS women had type 2 diabetes. ${ }^{29}$ Possible causes of not detecting any diabetes in the PCOS group could be due to no data on the oral glucose tolerance test (OGTT) to diagnose hyperglycemia, and the young age of the study participants. Although hyperinsulinemia is linked to PCOS in previous studies, ${ }^{2,4}$ the current data did not support such correlations. Such conflicting results could be due to the difference in the sample size, the study populations, and the heterogeneity of PCOS syndrome.

This study found that clinical risk factors that are significantly associated with PCOS are family history of PCOS and acne. A previous study by Azziz and Kashar-Miller ${ }^{30}$ noted that " $35 \%$ of mothers and $40 \%$ of sisters of patients with PCOS will be affected by PCOS themselves", which is consistent with the results of this study. Acne was present in $63.6 \%$ of PCOS subjects in this study. This is in line with previous studies showing that PCOS subjects had acne at a rate of $50 \%-80 \%$ in Turkey ${ }^{31}$ and Palestine. ${ }^{18}$ Among hormones, we found that DHEAS, testosterone, and SHBG are significantly associated with PCOS. A previous study by Asuncion et $\mathrm{al}^{17}$ in Spain demonstrated that DHEAS, testosterone, FAI, and SHBG are significant factors associated with PCOS. In this study, there were significantly higher testosterone (free and total), DHEAS, and FAI concentrations in PCOS subjects who had MI, which met the definition of PCOS, than in non-PCOS subjects (Table 3). The current data suggest that PCOS subjects with MI have higher levels of androgens than non-PCOS subjects, consistent with the definition of PCOS elsewhere. ${ }^{1,30}$

To the best of our knowledge, the association between this phenotype profile and the frequency of PCOS is the first of its kind to be gauged in this country. The current findings of phenotype profile and the frequency of PCOS of $18.33 \%$ cannot be used to extrapolate to the whole population of Qatar due to the low sample size, age distribution, and the selection confined to university students only. The strength of this study is that it is one of the first PCOS studies investigating the frequency and phenotypic profile of young females in Qatar. Using the international criteria by the NIH in this study, applicable data were drawn from the PCOS risk factors, frequency, and hormonal profile, so that further studies can be built on this data.

\section{Conclusion}

The frequency of PCOS among females aged 18-30 years in Qatar is found to be $18.33 \%$ using the NIH criteria. This study had several limitations; one of the most important was the limited sample size. Limited resources, such as ultrasound, at Qatar University clinic were an obstacle to evaluating the study subjects by another criterion, such as the Rotterdam criteria. $\mathrm{mFG}$ scoring was done by the participants themselves after demonstrations and instructions, which may have been influenced by social desirability bias.

Further studies should be conducted on a larger sample size. Longitudinal studies must be carried out to determine the long-term effects of PCOS on female health in Qatar.

\section{Disclosure}

The authors report no conflicts of interest in this work.

\section{References}

1. Azziz R. PCOS: a diagnostic challenge. Reprod Biomed Online. 2004; 8(6):644-648.

2. Balen A, Rajkowha M. Polycystic ovary syndrome - a systemic disorder? Best Pract Res Clin Obstet Gynaecol. 2003;17(2):263-274.

3. Bako AU, Morad S, Atiomo WA. Polycystic ovary syndrome: an overview. Rev Gynaecol Pract. 2005;5:115-122.

4. Diamanti-Kandarakis E, Christakou CD, Kandaraki E, Alexandraki KI. Early onset adiposity: a pathway to polycystic ovary syndrome in adolescents? Hormones (Athens). 2007;6(3):210-217.

5. Carmina E. Diagnosis of polycystic ovary syndrome: from NIH criteria to ESHRE-ASRM guidelines. Minerva Ginecol. 2004;56(1):1-6.

6. Geisthovel F. A comment on the European Society of Human Reproduction and Embryology/American Society for Reproductive Medicine consensus of the polycystic ovarian syndrome. Reprod Biomed Online. 2003;7(6):602-605.

7. Azziz R, Carmina E, Dewailly D, et al. The Androgen Excess and PCOS Society criteria for the polycystic ovary syndrome: the complete task force report. Fertil Steril. 2009;91(2):456-488.

8. Goodman NF, Bledsoe MB, Cobin RH, et al. American Association of Clinical Endocrinologists Hyperandrogenic Disorders Task F: American Association of Clinical Endocrinologists medical guidelines for the clinical practice for the diagnosis and treatment of hyperandrogenic disorders. Endocr Pract. 2001;7(2):120-134.

9. Martin KA, Chang RJ, Ehrmann DA, et al. Evaluation and treatment of hirsutism in premenopausal women: an endocrine society clinical practice guideline. J Clin Endocrinol Metab. 2008;93(4):1105-1120. 
10. Al-Ruhaily AD, Malabu UH, Sulimani RA. Hirsutism in Saudi females of reproductive age: a hospital-based study. Ann Saudi Med. 2008;28(1): 28-32.

11. Vermeulen A, Verdonck L, Kaufman JM. A critical evaluation of simple methods for the estimation of free testosterone in serum. J Clin Endocrinol Metab. 1999;84(10):3666-3672.

12. Rosner W, Auchus RJ, Azziz R, Sluss PM, Raff H. Position statement: utility, limitations, and pitfalls in measuring testosterone: an Endocrine Society position statement. J Clin Endocrinol Metab. 2007;92(2): 405-413.

13. Tehrani FR, Simbar M, Tohidi M, Hosseinpanah F, Azizi F. The prevalence of polycystic ovary syndrome in a community sample of Iranian population: Iranian PCOS prevalence study. Reprod Biol Endocrinol. 2011;9:39

14. Mehrabian F, Khani B, Kelishadi R, Ghanbari E. The prevalence of polycystic ovary syndrome in Iranian women based on different diagnostic criteria. Endokrynol Pol. 2011;62(3):238-242.

15. Kumarapeli V, Seneviratne Rde A, Wijeyaratne CN, Yapa RM, Dodampahala SH. A simple screening approach for assessing community prevalence and phenotype of polycystic ovary syndrome in a semi-urban population in Sri Lanka. Am J Epidemiol. 2008;168(3):321-328.

16. Wijeyaratne CN, Seneviratne Rde A, Dahanayake S, et al. Phenotype and metabolic profile of South Asian women with polycystic ovary syndrome (PCOS): results of a large database from a specialist Endocrine Clinic. Hum Reprod. 2011;26(1):202-213.

17. Asuncion M, Calvo RM, San Millan JL, Sancho J, Avila S, EscobarMorreale HF. A prospective study of the prevalence of the polycystic ovary syndrome in unselected Caucasian women from Spain. J Clin Endocrinol Metab. 2000;85(7):2434-2438.

18. Musmar S, Afaneh A, Mo'alla H. Epidemiology of polycystic ovary syndrome: a cross sectional study of university students at An-Najah National university-Palestine. Reprod Biol Endocrinol. 2013;11:47.

19. Rey-Roldan E, Perez Lana MB, Galluzzo L, et al. Is the polycystic ovary syndrome the causative of the increase in inflammatory markers and metabolic risk? Gynecol Endocrinol. 2013;29(2):141-144.
20. Lowe P, Kovacs G, Howlett D. Incidence of polycystic ovaries and polycystic ovary syndrome amongst women in Melbourne, Australia. Aust N Z J Obstet Gynaecol. 2005;45(1):17-19.

21. Gomathi K, Shaafie I, Mummigatti K, Shahid S, Sreedharan J. Biochemical Parameters in Women with Polycystic Ovary Syndrome in Ajman, UAE. Nepal J Obstet Gynaecol. 2012;6(2):7-10.

22. Knochenhauer ES, Key TJ, Kahsar-Miller M, Waggoner W, Boots LR, Azziz R. Prevalence of the polycystic ovary syndrome in unselected black and white women of the southeastern United States: a prospective study. J Clin Endocrinol Metab. 1998;83(9):3078-3082.

23. Huang A, Brennan K, Azziz R. Prevalence of hyperandrogenemia in the polycystic ovary syndrome diagnosed by the National Institutes of Health 1990 criteria. Fertil Steril. 2010;93(6):1938-1941.

24. Gatee OB, Al Attia HM, Salama IA. Hirsutism in the United Arab Emirates: a hospital study. Postgrad Med J. 1996;72(845):168-171.

25. March WA, Moore VM, Willson KJ, Phillips DI, Norman RJ, Davies MJ. The prevalence of polycystic ovary syndrome in a community sample assessed under contrasting diagnostic criteria. Hum Reprod. 2010;25(2): 544-551.

26. Whitaker KN. Polycystic ovary syndrome: an overview. J Pharm Pract. 2011;24(1):94-101.

27. Kovács JTNR. Polycystic Ovary Syndrome. 2nd ed. Cambridge: Cambridge University Press; 2007.

28. Liang SJ, Hsu CS, Tzeng CR, Chen CH, Hsu MI. Clinical and biochemical presentation of polycystic ovary syndrome in women between the ages of 20 and 40. Hum Reprod. 2011;26(12):3443-3449.

29. Buccola JM, Reynolds EE. Polycystic ovary syndrome: a review for primary providers. Prim Care . 2003;30(4):697-710.

30. Azziz R, Kashar-Miller MD. Family history as a risk factor for the polycystic ovary syndrome. J Pediatr Endocrinol Metab. 2000; 13(suppl 5):1303-1306.

31. Chang WY, Knochenhauer ES, Bartolucci AA, Azziz R. Phenotypic spectrum of polycystic ovary syndrome: clinical and biochemical characterization of the three major clinical subgroups. Fertil Steril. 2005;83(6):1717-1723. 


\section{Supplementary materials}

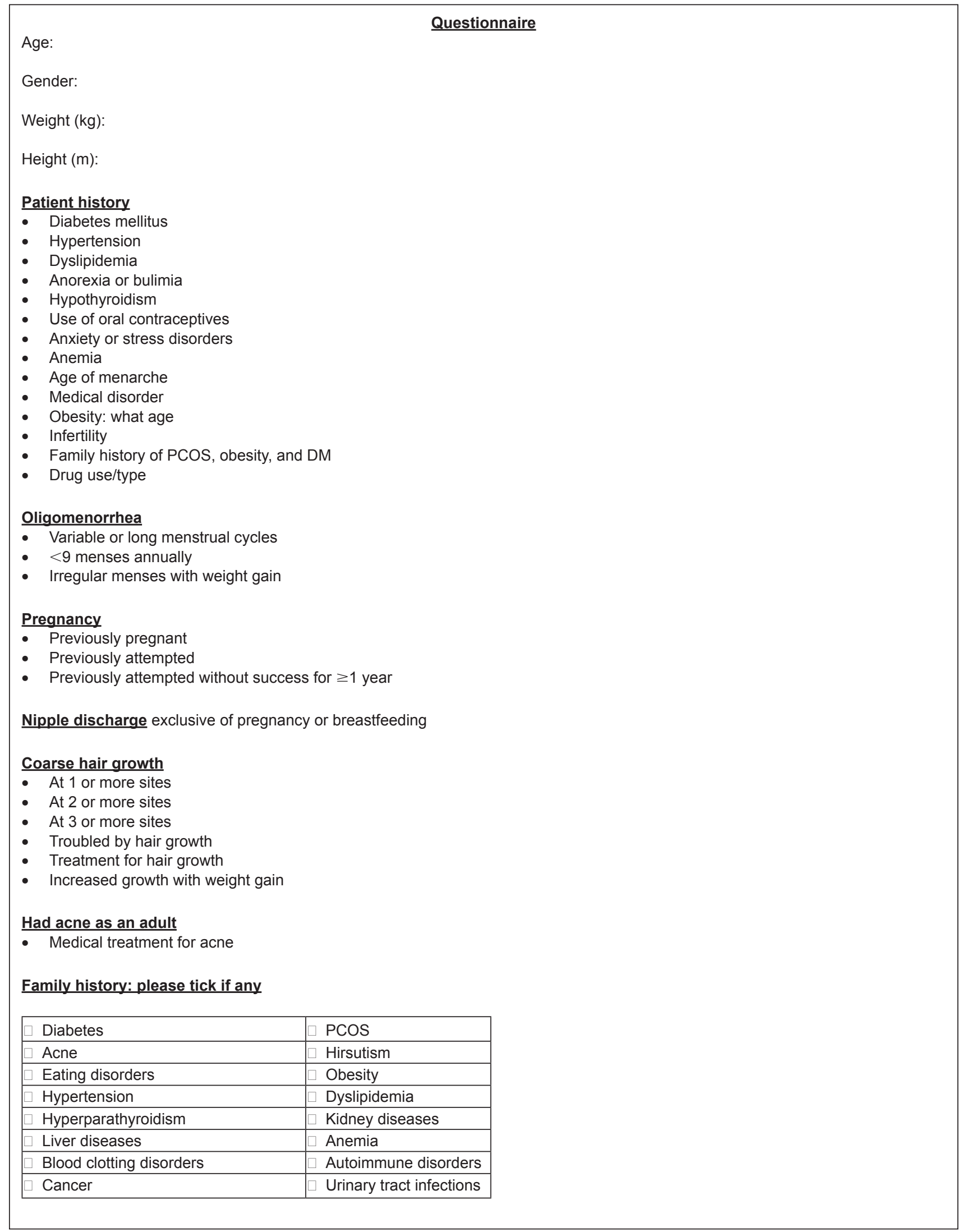

Figure SI (Continued) 


\section{Please tick if you have one of the following complaints:}

\begin{tabular}{|l|l|}
\hline Complaint & Please tick if any = yes \\
\hline Mood disorders & \\
\hline Anxiety & \\
\hline Bulimia & \\
\hline Nausea & \\
\hline Vomiting & \\
\hline Anorexia & \\
\hline Weight changes & \\
\hline Constipation & \\
\hline Diarrhea & \\
\hline Dermatological problem & \\
\hline
\end{tabular}

\section{Life style changes:}

Gain weight

Food: type and quantity

Appetite

Mood changes

Relationship with others

Figure SI Patient questionnaire.

Abbreviations: DM, diabetes mellitus; PCOS, polycystic ovary syndrome.

Table SI Cutoff values for hormones used by Hamad Medical Corporation, Qatar

\begin{tabular}{lc}
\hline Hormone & Cutoff value \\
\hline Prolactin & $109-557 \mathrm{mlU} / \mathrm{L}$ \\
Testosterone & $0.69-2.78 \mathrm{nmol} / \mathrm{L}$ \\
DHEAS & $3.6-1 \mathrm{I} .10 \mu \mathrm{mol} / \mathrm{L}$ \\
SHBG & $20-100 \mathrm{nmol} / \mathrm{L}$ \\
Insulin & $1.9-23 \mathrm{mlU} / \mathrm{L}$ \\
Glucose & $105 \mathrm{mg} / \mathrm{dL}$ \\
TSH & $0.45-4.5 \mathrm{mlU} / \mathrm{L}$ \\
\hline
\end{tabular}

Abbreviations: DHEAS, dehydroepiandrosterone sulfate; SHBG, sex hormone-binding globulin; TSH, thyroid-stimulating hormone.

Table S2 Participants who met the individual NIH diagnostic criteria for PCOS ( $n=22,18.33 \%)$

\begin{tabular}{lc}
\hline Criteria & $\mathbf{n}(\%)$ \\
\hline Clinical or biochemical hyperandrogenism, $\mathrm{n}(\%)$ & $22(100.0)$ \\
Hirsutism & $22(100.0)$ \\
Clinical hyperandrogenism (\%) & $22(100)$ \\
Acne & $14(63.30)$ \\
Increased free testosterone & $0.0(0.0)$ \\
Increased FAl & $19(86.36)$ \\
Biochemical hyperandrogenism (\%) & $19(86.36)$ \\
Oligoovulation & $22(100.0)$ \\
Chronic oligomenorrhea and/or amenorrhea & $22(100.0)$ \\
Menstrual irregularity + clinical hyperandrogenism & $22(100)$ \\
Menstrual irregularity + biochemical hyperandrogenism & $19(86.36)$ \\
Exclusion of secondary causes & $22(100)$ \\
\hline
\end{tabular}

Note: Values are raw numbers (percentage).

Abbreviations: FAl, free androgen index; NIH, US National Institutes of Health; PCOS, polycystic ovary syndrome. 
International Journal of Women's Health

Dovepress

\section{Publish your work in this journal}

The International Journal of Women's Health is an international, peerreviewed open-access journal publishing original research, reports, editorials, reviews and commentaries on all aspects of women's healthcare including gynecology, obstetrics, and breast cancer. The manuscript management system is completely online and includes Visit http://www.dovepress.com/testimonials.php to read real quotes from published authors.

Submit your manuscript here: http://www.dovepress.com/international-journal-of-womens-health-journal 